\title{
Tissue kallikrein promotes survival and $\beta$-catenin degradation in SH-SY5Y cells under nutrient stress conditions via autophagy
}

\author{
YANPING LIU ${ }^{1}$, MEI CUI ${ }^{1}$, ZHENGYU LU ${ }^{2}$, QI YANG ${ }^{1}$ and QIANG DONG ${ }^{1}$ \\ ${ }^{1}$ Department of Neurology, Huashan Hospital, State Key Laboratory of Medical Neurobiology, Fudan University, \\ Shanghai 200040; ${ }^{2}$ Department of Neurology, Yueyang Hospital of Integrated Traditional Chinese and Western Medicine, \\ Shanghai University of Traditional Chinese Medicine, Shanghai 200437, P.R. China
}

Received February 1, 2015; Accepted November 19, 2015

DOI: $10.3892 / \mathrm{mmr} .2015 .4664$

\begin{abstract}
Previous studies by our group showed that tissue kallikrein (TK) exerts neuroprotective effects during cerebral ischemia. Autophagy is an important adaptive response to cellular stress during nutrient deprivation, and $\beta$-catenin in known to repress autophagy. The present study investigated the possible involvement of autophagy and $\beta$-catenin signaling in the protective effects of TK under nutrient deprivation-induced stress conditions. TK was shown to promote the survival and inhibit the death of SH-SY5Y cells under serum starvation and enhanced autophagic activity in a concentration-dependent manner, as indicated by augmented light chain (LC)3-II levels and Beclin-1 expression. The autophagy inhibitors 3-methyladenine and $\mathrm{NH}_{4} \mathrm{Cl}$ abolished the protective effects of TK. Of note, although serum starvation alone and TK treatment increased p62 protein levels and mRNA expression, incubation with the lysosome inhibitor $\mathrm{NH}_{4} \mathrm{Cl}$ increased the accumulation of LC3-II and p62 protein, indicating normal autophagic flux. It was also observed that $\beta$-catenin expression was significantly downregulated by TK treatment. TK stimulated the interaction between $\mathrm{LC} 3$ and $\beta$-catenin, and $\mathrm{NH}_{4} \mathrm{Cl}$ abolished the effects of TK on $\beta$-catenin levels in serum-starved cells, suggesting the autophagic degradation of $\beta$-catenin, which may have led to the enhancement of autophagy. In conclusion, the findings of the present study demonstrated that TK promoted cell survival and $\beta$-catenin degradation in serum-starved SH-SY5Y cells via increasing autophagy, which indicated the therapeutic potential of TK under nutrient deprivation-associated stress conditions.
\end{abstract}

Correspondence to: Professor Qiang Dong, Department of Neurology, Huashan Hospital, State Key Laboratory of Medical Neurobiology, Fudan University, 12 Mid Wulumuqi Road, Shanghai 200040, P.R. China

E-mail: dong_qiang@fudan.edu.cn

Key words: tissue kallikrein, cell survival, starvation, autophagy, $\beta$-catenin

\section{Introduction}

Autophagy is an adaptive response to cellular stress induced by conditions such as nutrient deprivation. During autophagy, cellular components to be degraded are surrounded by autophagosomes and catabolized after fusion with lysosomes to provide precursors for synthetic processes and energy production, which contributes to cellular survival (1).

Tissue kallikrein (TK), an important component of the kallikrein-kinin system, belongs to a sub-group of serine proteinases and processes low-molecular-weight kininogen to release kinin peptide (2). Previous studies by our group showed that TK exerted neuroprotective effects in oxygen-glucose-deprived cells as well as in the ischemic brain (3-5), in which autophagy has been reported to have an important role in maintaining neuronal survival (6). It has been reported that TK is able to activate the Raf/mitogen-activated protein kinase kinase/extracellular signal-regulated kinase pathway (4) and adenosine 5'-monophosphate-activated protein kinase phosphorylation (7), which participate in autophagy induction $(8,9)$. However, to date, no direct evidence has revealed whether autophagy is involved in TK-mediated protective effects under nutrient deprivation-induced stress conditions.

$\beta$-catenin is a key downstream effector in the Wnt signaling pathway. The activation of the $\mathrm{Wnt} / \beta$-catenin axis is known to increase cell survival and proliferation under various conditions (10). A recent study demonstrated that activated $\beta$-catenin signaling was able to repress autophagy. However, $\beta$-catenin protein contains a light chain (LC)3-interacting motif, which is targeted for autophagic clearance, thus facilitating autophagy induction and cell survival under nutrient deficiency (11).

The present study used serum-starved SH-SY5Y human neuroblastoma cells as a canonical model for investigating autophagy. It was attempted to evaluate the role of TK in modulating autophagy and its crosstalk with $\beta$-catenin signaling under nutrient deprivation-induced stress conditions. It was indicated that TK promoted the induction of protective autophagy along with autophagic degradation of $\beta$-catenin and maintained normal autophagic flux. 


\section{Materials and methods}

Reagents. TK was purchased from Techpool Bio-Pharma Co. (Guangzhou, China). Rabbit anti-human LC3A/B (cat no. 12741), LC3B (cat no. 3868) and Beclin-1 (cat. no. 3495) as well as mouse anti-human $\alpha$-tubulin (cat. no. 3873) monoclonal antibodies were obtained from Cell Signaling Technology, Inc. (Danvers, MA, USA). Mouse anti-human sequestosome 1 (p62; cat. no. ab56416) and rabbit anti-human $\beta$-catenin (cat. no. ab32572) monoclonal antibodies were purchased from Abcam (Cambridge, MA, USA). Horseradish peroxidase (HRP)-conjugated goat anti-rabbit immunoglobulin (Ig)G (cat. no. GGHL-15P) and HRP-conjugated goat anti-mouse IgG (cat. no. GGHL-90P) secondary antibodies were obtained from Immunology Consultants Laboratory (Portland, OR, USA). 3-(4,5-dimethylthiazol-2-yl)-2,5-diphenyl tetrazolium bromide (MTT), 3-methyladenine (3-MA) and $\mathrm{NH}_{4} \mathrm{Cl}$ were obtained from Sigma-Aldrich (St. Louis, MO, USA).

Cell culture. Human SH-SY5Y neuroblastoma cells were purchased from the American Type Culture Collection (Manassas, VA, USA). Cells were grown in Dulbecco's modified Eagle's medium supplemented with $10 \%$ fetal bovine serum (Invitrogen; Thermo Fisher Scientific, Waltham, MA, USA) supplemented with $100 \mathrm{U} / \mathrm{ml}$ penicillin and $100 \mu \mathrm{g} / \mathrm{ml}$ streptomycin (Invitrogen) at $37^{\circ} \mathrm{C}$ in a humidified atmosphere containing $5 \% \mathrm{CO}_{2}$. Cells were grown to $80 \%$ confluency prior to the experiments.

Cell viability and apoptosis assays. SH-SY5Y cells were seeded into 96-well plates at $2 \times 10^{4}$ cells per well and allowed to adhere overnight. Cells were treated with indicated concentrations of TK $(0,0.125,0.25,0.5$ and $1 \mu \mathrm{M})$ followed by serum starvation for 12 or $24 \mathrm{~h}$. Subsequently, $10 \mu \mathrm{l}$ MTT solution $(5 \mathrm{mg} / \mathrm{ml})$ was added to each well, followed by incubation at $37^{\circ} \mathrm{C}$ for $4 \mathrm{~h}$ in the dark. Following removal of the MTT solution and addition of $150 \mu \mathrm{l}$ dimethyl sulfoxide (Sigma-Aldrich) to dissolve the formazan crystals, the absorbance at $570 \mathrm{~nm}$ was measured using a plate reader (Synergy H1, BioTek Instruments, Winooski, VT, USA). The cell viability was determined by calculating the absorbance ratio of treated cells vs. that of unstarved control cells (viability, 100\%).

For the apoptosis assay, cells were treated as stated above, harvested and stained using the FITC Annexin V Apoptosis Detection Kit I (BD Pharmingen, San Jose, CA, USA) according to the manufacturer's instructions and subsequently analyzed using flow cytometry (BD FACSCanto; BD Pharmingen) with BD FACSDiva software (BD Pharmingen). Quantitative analysis of cell death by flow cytometry Annexin V/PI staining. Dead cells included early and late apoptotic cells (lower and upper right quadrants in dot plot) as well as necrotic cells (upper left quadrant).

Co-immunoprecipitation. Co-immunoprecipitation was performed according to the manufacturer's instructions of the protein A/G PLUS-Agarose Immunoprecipitation Reagent (Santa Cruz Biotechnology, Inc., Dallas, TX, USA). Briefly, SH-SY5Y cells were lysed on ice in $1 \mathrm{ml}$ radioimmunoprecipitation assay (RIPA) lysis buffer (Beyotime Institute of Biotechnology, Haimen, China) for $30 \mathrm{~min}$.
Following pre-clearing with normal $\operatorname{IgG}$ (Beyotime Institute of Biotechnology), cell lysates were incubated overnight at $4^{\circ} \mathrm{C}$ with anti-LC3B (diluted at 1:50), followed by precipitation with $20 \mu 1$ protein A/G Plus-Agarose (Santa Cruz Biotechnology, Inc.) overnight at $4^{\circ} \mathrm{C}$. The precipitated complexes were washed four times with phosphate-buffered saline (PBS) and heated at $100^{\circ} \mathrm{C}$ for $5 \mathrm{~min}$ in $5 \mathrm{X}$ sodium dodecyl sulfate (SDS) sample buffer (Beyotime Institute of Biotechnology). The samples were then separated on 10\% SDS-polyacrylamide gels and immunoblotted with $\beta$-catenin monoclonal antibody overnight at $4^{\circ} \mathrm{C}$. Membranes were routinely washed four times in Tris-buffered saline with $0.1 \%$ Tween 20 (TBST) for $5 \mathrm{~min}$ and then incubated with HRP-conjugated goat anti-rabbit IgG for $1 \mathrm{~h}$ at room temperature. The antigen-antibody complexes were visualized using the Immobilon ${ }^{\mathrm{TM}}$ western chemiluminescent HRP substrate (Millipore, Billerica, MA, USA) and images were captured using the ChemiDoc XRS+ system (Bio-Rad Laboratories, Hercules, CA, USA).

Western blot analysis. $2 \times 10^{7} \mathrm{SH}-\mathrm{SY} 5 \mathrm{Y}$ cells were lysed on ice and washed with ice-cold PBS and lysed on ice for $20 \mathrm{~min}$ with RIPA lysis buffer supplemented with protease inhibitors (Roche Diagnostics, Basel, Switzerland). Protein concentrations were determined using a bicinchoninic acid assay kit (Beyotime Institute of Biotechnology). Equal amounts of protein $(20 \mu \mathrm{g})$ were separated by electrophoresis on SDS-polyacrylamide gels [prepared with $30 \%$ acrylamide-bisacrylamide, $1 \mathrm{M}$ Tris- $\mathrm{HCl}$ (pH 6.8 and $\mathrm{pH} 8.8$ ), ammonium persulfate and tetramethylethylenediamine; all from Beyotime Institute of Biotechnology) and transferred to an Immobilon-P polyvinylidene difluoride membrane (Millipore). Immunoblotting was performed with the appropriate primary antibody at 1:1,000 dilution overnight at $4^{\circ} \mathrm{C}$, followed by HRP-conjugated secondary antibody at 1:5,000 dilution for $1 \mathrm{~h}$ at room temperature. Blotted proteins were visualized using enhanced chemiluminescence (using the Immobilon ${ }^{\mathrm{TM}}$ western chemiluminescent HRP substrate) and images were captured using the ChemiDoc XRS+ system (Bio-Rad Laboratories). Densitometric analysis was performed using Image J 1.4.3.67 software (National Institutes of Health, Bethesda, MD, USA) with $\alpha$-tubulin as the loading control. Relative density values to $\alpha$-tubulin for each sample were normalized to the basal levels in the control group to obtain fold-change values.

Reverse-transcription quantitative polymerase chain reaction $(R T-q P C R)$. Following treatment as stated above, total RNA was extracted using TRIzol reagent (Invitrogen) according to the manufacturer's instructions. Complementary DNA was synthesized using the PrimeScript ${ }^{\mathrm{TM}}$ RT Master Mix (no. RR036A; Takara Bio Inc., Otsu, Japan). qPCR was performed using a SYBR Green PCR kit (no. DRR041A; Takara Bio Inc.) in an ABI real-time PCR system (Applied Biosystems; Thermo Fisher Scientific) according to the manufacturer's instructions. The following primers (Sangon Biotech, Shanghai, China) were used: p62 forward, 5'-AAATGGGTCCACCAGGAA ACTGGA-3' and reverse, 5'-TCAACTTCAATGCCCAGA GGGCTA-3'; GAPDH forward, 5'-CTGGGCTACACTGAG CACC-3' and reverse, 5'AAGTGGTCGTTGAGGGCAATG-3'. The thermocycling conditions were as follows: Polymerase activation/denaturation $\left(95^{\circ} \mathrm{C}\right.$ for $\left.30 \mathrm{sec}\right)$ and 45 amplification cycles 
A

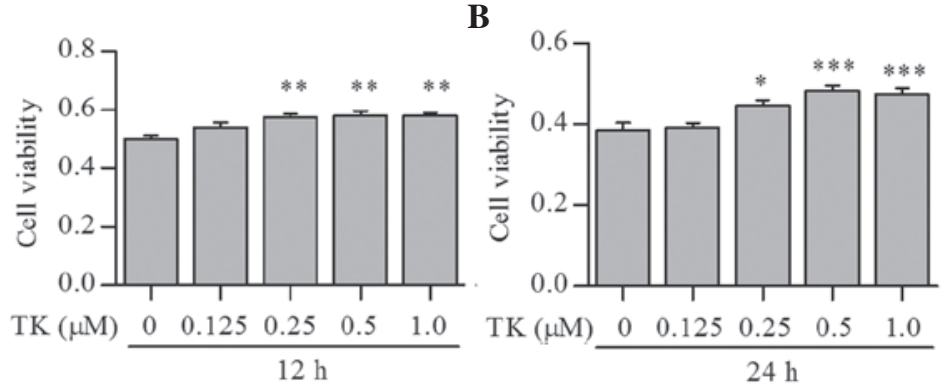

C

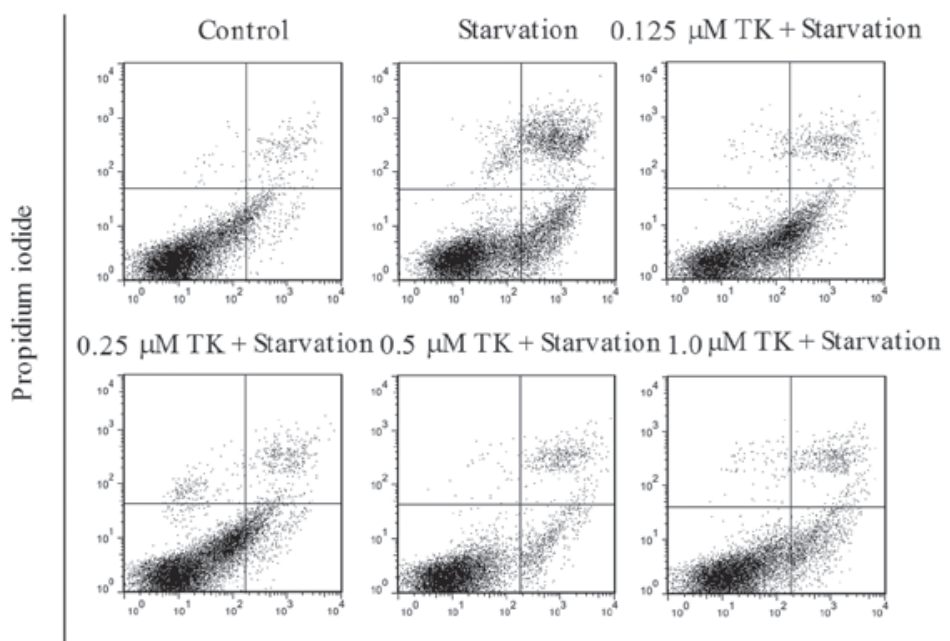

D

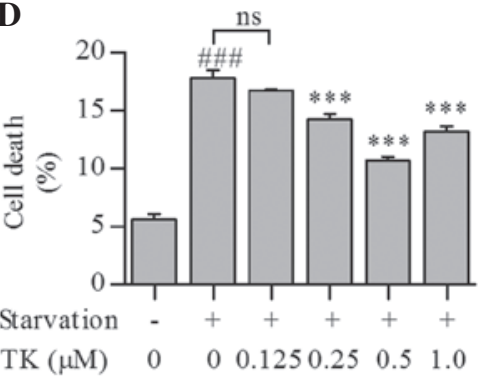

FITC-Annexin V

Figure 1. TK promotes survival of SH-SY5Y cells under serum starvation. (A and B) SH-SY5Y cells were pre-treated with $0.125-1.0 \mu \mathrm{M}$ TK followed by serum starvation for (A) $12 \mathrm{~h}$ or (B) $24 \mathrm{~h}$, and the cell viability was examined using an MTT assay. (C) Representative flow cytometry dot plots of Annexin V/propidium iodide-stained SH-SY5Y cells pre-treated with 0.125-1.0 $\mu \mathrm{M}$ TK and serum-starved for $24 \mathrm{~h}$. (D) The apoptotic rate was determined by quantification of Annexin V and propidium iodide staining. Dead cells included early and late apoptotic cells (lower and upper right quadrants in dot plot) as well as necrotic cells (upper left quadrant)..Values are expressed as the mean \pm standard deviation of at least three individual experiments. ns, not significant; ${ }^{\# \# ~} \mathrm{P}<0.001 \mathrm{vs}$. no starvation; ${ }^{*} \mathrm{P}<0.05,{ }^{* *} \mathrm{P}<0.01,{ }^{* * * *} \mathrm{P}<0.001$ vs. serum-starved group not treated with TK. FITC, fluorescein isothiocyanate; TK, tissue kallikrein.

$\left(95^{\circ} \mathrm{C}\right.$ for $5 \mathrm{sec}, 58^{\circ} \mathrm{C}$ for $30 \mathrm{sec}$ and $72^{\circ} \mathrm{C}$ for $30 \mathrm{sec}$ ). Relative p62 mRNA levels were normalized to GAPDH and calculated using the $2^{-\Delta \Delta \mathrm{Cq}}$ method. $\Delta \mathrm{Cq}$ was calculated by subtracting their $\mathrm{Cq}$ values from the average $\mathrm{Cq}$ value of $\mathrm{GAPDH}$.

Statistical analysis. Values are expressed as the mean \pm standard deviation. Differences were evaluated by one-way analysis of variance followed by Bonferroni's post-hoc test with GraphPad Prism 5 software (GraphPad Inc., La Jolla, CA, USA). P<0.05 was considered to indicate a statistically significant difference between values.

\section{Results}

TK promotes survival of SH-SY5Y cells under serum starvation. To determine whether TK is able to promote cell survival under nutrient deprivation-induced stress conditions, SH-SY5Y cells were treated with TK at $0.125-1.0 \mu \mathrm{M}$ and then subjected to serum starvation for 12 or $24 \mathrm{~h}$. As shown in Fig. 1A and B, TK augmented the viability of SH-SY5Y cells in a time- and concentration-dependent manner, with $0.5 \mu \mathrm{M}$ of TK displaying the most significant protective effects at $24 \mathrm{~h}$. Annexin $\mathrm{V}$ and propidium iodide staining also showed that cell death induced by starvation for $24 \mathrm{~h}$ was partly prevented by TK at $0.25-1.0 \mu \mathrm{M}$ (Fig. 1C and D).
Pro-survival effects of TK are based on the induction of autophagy. To explore whether autophagic pathways were involved in the pro-survival effects of TK, LC3 conversion and Beclin-1 expression were assessed as autophagic markers in serum-starved SH-SY5Y cells pre-treated with TK. Compared to serum starvation alone, TK concentration-dependently induced a more profound elevation of LC3-II and expression of Beclin-1 (Fig. 2A-C), with the most significant effects at 0.5 and $1.0 \mu \mathrm{M}$.

In an additional experiment, SH-SY5Y cells were pre-treated with $0.5 \mu \mathrm{M}$ TK in combination with the autophagy inhibitors 3-MA or $\mathrm{NH}_{4} \mathrm{Cl}$ (12), followed by serum starvation for $24 \mathrm{~h}$ and detection of cell viability using an MTT assay. Pre-treatment with 3-MA or $\mathrm{NH}_{4} \mathrm{Cl}$ alone diminished the viability of SH-SY5Y cells under serum starvation. Furthermore, the protective effects of TK were abolished by 3-MA as well as $\mathrm{NH}_{4} \mathrm{Cl}$ (Fig. 2D). These results suggested that autophagy induction contributed to the pro-survival effects of TK.

TK upregulates p62 expression while maintaining normal autophagic flux in serum-starved SH-SY5Y cells. p62 is known to scavenge soluble mis-folded proteins for removal in autophagosomes (1) and can be used as a protein marker of autophagic degradation and autophagic flux in certain 
A

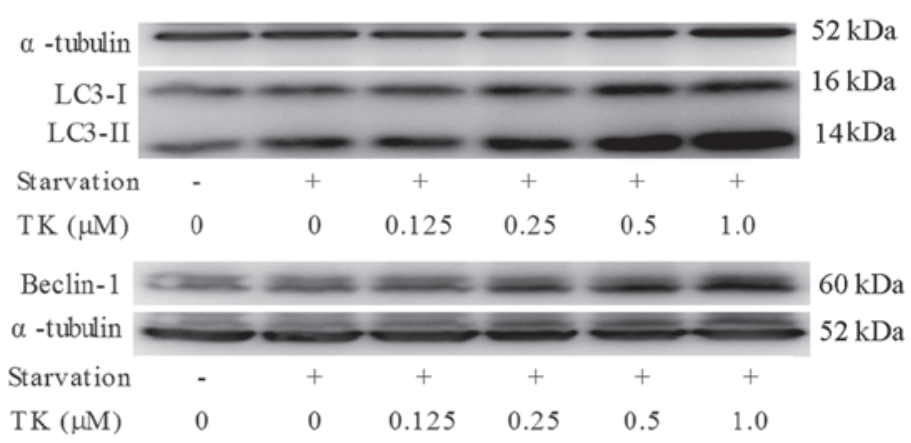

C

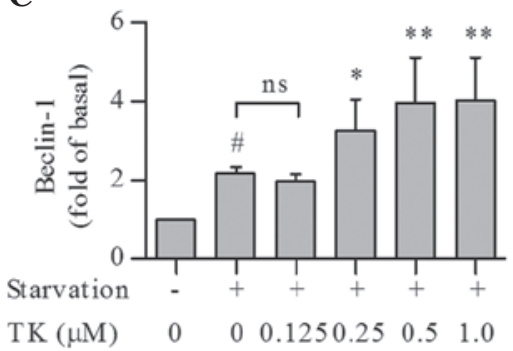

B

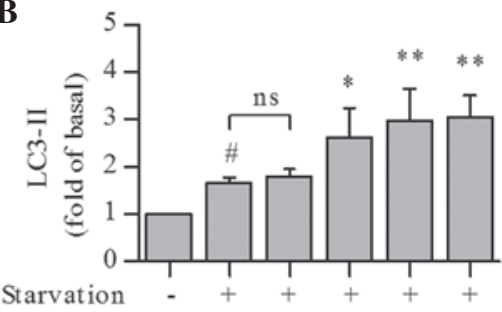

$\mathrm{TK}(\mu \mathrm{M}) \quad 0 \quad 0 \quad 0.125 \quad 0.25 \quad 0.5 \quad 1.0$

D

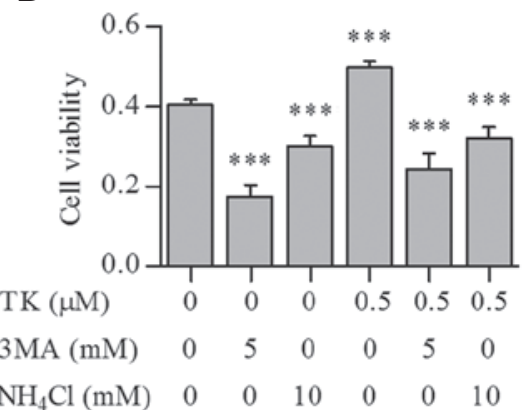

Figure 2. Autophagy contributes to TK-mediated pro-survival effects in serum-starved SH-SY5Y cells. (A-C) SH-SY5Y cells were pre-treated with 0.125-1.0 $\mu \mathrm{M}$ TK and serum-starved for $12 \mathrm{~h}$. LC3 conversion and Beclin-1 levels were examined by western blot analysis with $\alpha$-tubulin used as the loading control. (D) SH-SY5Y cells were pre-treated with $5 \mathrm{mM} 3-\mathrm{MA}$ or $10 \mathrm{mM} \mathrm{NH}_{4} \mathrm{Cl}$ or $0.5 \mu \mathrm{M} \mathrm{TK}$ in combination with 3 - $\mathrm{MA}_{\text {or }} \mathrm{NH}_{4} \mathrm{Cl}$ and serum-starved for 24 h. Cell viability was examined using an MTT assay. Values are expressed as the mean \pm standard deviation of at least three individual experiments. ns, not significant; ${ }^{\#} \mathrm{P}<0.05$ vs. no starvation; ${ }^{*} \mathrm{P}<0.05,{ }^{* *} \mathrm{P}<0.01,{ }^{* * *} \mathrm{P}<0.001$ vs. serum-starved group not treated with any drugs. TK, tissue kallikrein; LC, light chain; MA, methyladenine.

settings (12). Unexpectedly, p62 protein levels were significantly increased by serum starvation and further increased by TK. TK elevated the protein levels of p62 in a concentration-dependent manner, with the most significant changes at $0.5 \mu \mathrm{M}$ (Fig. 3A). Similarly to its effect on p62 protein levels, TK at $0.25-1.0 \mu \mathrm{M}$ also promoted the transcription of $\mathrm{p} 62$ as compared to that following serum starvation alone, as indicated by RT-qPCR analysis (Fig. 3B). This result indicated that increases in p62 protein were due to enhanced transcription, and did not reflect autophagic flux in this scenario. To further assess autophagic flux, cells were pre-treated with $0.5 \mu \mathrm{M}$ TK in combination with $\mathrm{NH}_{4} \mathrm{Cl}$, an inhibitor of lysosomal acidification that can inhibit autophagic degradation (12), followed by serum starvation. $\mathrm{NH}_{4} \mathrm{Cl}$ significantly raised the protein levels of p62 in serum-starved SH-SY5Y cells at $12 \mathrm{~h}$, irrespective of the presence of TK (Fig. 3C), indicating that p62 was indeed degraded in the autophagic process. In addition, the effects of $\mathrm{NH}_{4} \mathrm{Cl}$ on LC3-II levels were assessed at $6 \mathrm{~h}$ (Fig. 3D) and $12 \mathrm{~h}$ (Fig. 3E), which revealed that $\mathrm{NH}_{4} \mathrm{Cl}$ led to the accumulation of LC3-II at each time-point with or without pre-treatment with TK. These results confirmed that TK maintained a normal autophagic flux.

TK stimulates the autophagic degradation of $\beta$-catenin. It was previously reported that $\beta$-catenin represses autophagy but is also degraded during the autophagic process (11). Therefore, $\beta$-catenin levels in serum-starved SH-SY5Y cells treated in the presence or absence of TK were assessed. $\beta$-catenin expression remained unchanged following serum starvation for $12 \mathrm{~h}$ as compared to that in the control cells. While $0.125 \mu \mathrm{M}$ TK led to an upregulation of $\beta$-catenin expression, TK at $0.25-1.0 \mu \mathrm{M}$ significantly decreased the levels of $\beta$-catenin, with $0.5 \mu \mathrm{M}$ TK causing the most significant reduction of $\beta$-catenin (Fig. 4A).

To further investigate whether downregulation of $\beta$-catenin by TK was due to autophagic degradation, $\beta$-catenin levels were examined in serum-starved SH-SY5Y cells pre-treated with $0.5 \mu \mathrm{M}$ TK in combination with $\mathrm{NH}_{4} \mathrm{Cl}$. $\mathrm{NH}_{4} \mathrm{Cl}$ abolished the effects of $0.5 \mu \mathrm{M}$ TK on $\beta$-catenin levels in serum-starved SH-SY5Y cells, while treatment with $\mathrm{NH}_{4} \mathrm{Cl}$ alone had no significant impact on $\beta$-catenin levels in serum-starved cells (Fig. 4B). Co-immunoprecipitation analysis then revealed that TK induced an interaction between LC3 and $\beta$-catenin in serum-starved SH-SY5Y cells (Fig. 4C), suggesting that TK stimulated the autophagic degradation of $\beta$-catenin.

\section{Discussion}

TK has been reported to have important roles in various physiological and pathological processes $(13,14)$. The present study revealed that TK enhanced the survival of human SH-SY5Y cells under serum starvation in a starvation timeand TK concentration-dependent manner. Previous studies demonstrated that TK transactivates multiple downstream signaling pathways, including the phosphoinositide-3 kinase (PI3K)/Akt and epidermal growth factor receptor (EGFR) signaling $(3,15,16)$. PI3K/Akt activation and EGFR are able to activate $\mathrm{Wnt} / \beta$-catenin signaling but suppress autophagy induction (17-20). However, the present study revealed that $\beta$-catenin expression was significantly downregulated by TK treatment, accompanied by increased autophagy, suggesting that the underlying molecular mechanism is independent of the PI3K/Akt and EGFR pathways. 
A
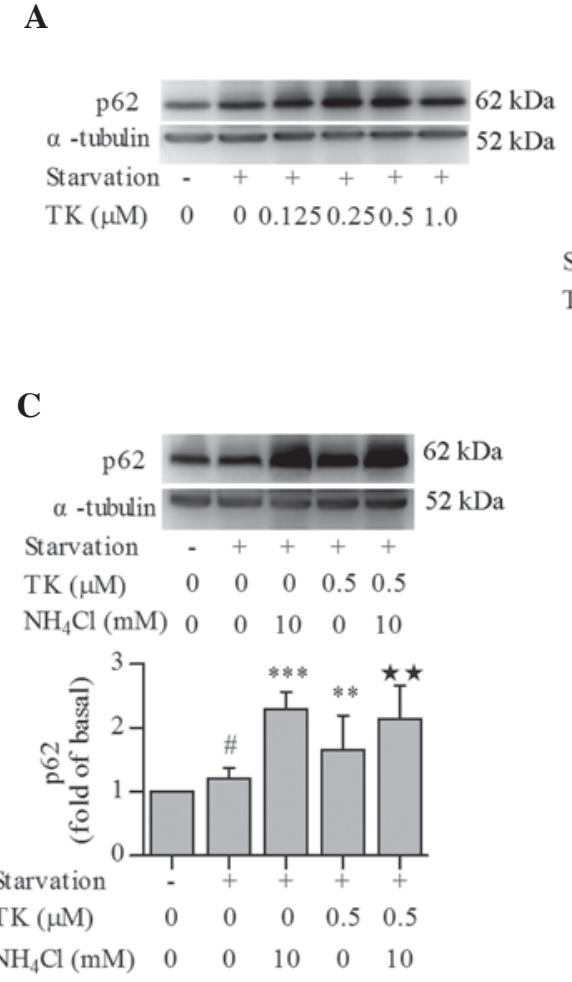
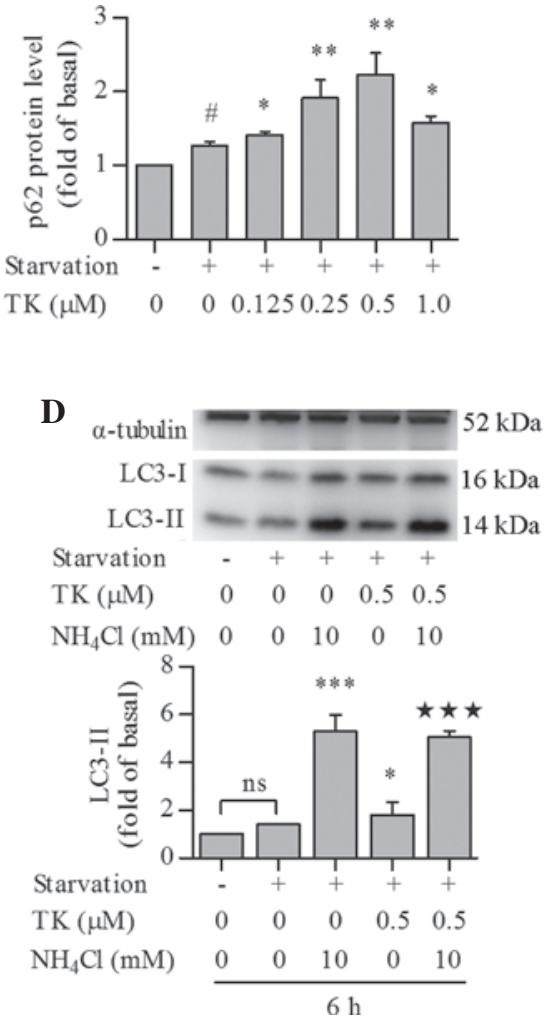

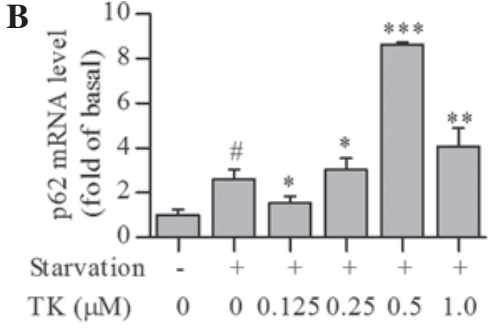

$\mathbf{E}$
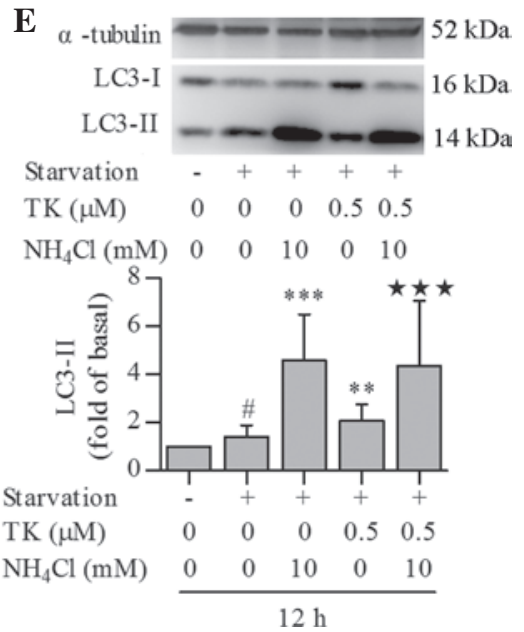

Figure 3. TK upregulates p62 expression, while maintaining normal autophagic flux in serum-starved SH-SY5Y cells. (A) SH-SY5Y cells were pre-treated with 0.125-1.0 $\mu \mathrm{M}$ TK and serum-starved for $12 \mathrm{~h}$. p62 protein levels were examined by western blot analysis. $\alpha$-tubulin was used as the loading control. (B) SH-SY5Y cells were treated with 0.125-1.0 $\mu \mathrm{M}$ TK and serum-starved for $8 \mathrm{~h} . \mathrm{p} 62 \mathrm{mRNA}$ levels were examined by quantitative polymerase chain reaction. (C-E) SH-SY5Y cells were treated with $0.5 \mu \mathrm{M} \mathrm{TK}$ or in combination with $10 \mathrm{mM} \mathrm{NH}_{4} \mathrm{Cl}$ and serum-starved for 6 or $12 \mathrm{~h}$. (C) p62 levels at $12 \mathrm{~h}$, (D) LC3 levels at $6 \mathrm{~h}$ and (E) LC3 levels at $12 \mathrm{~h}$ were detected by western blot. $\alpha$-tubulin was used as the loading control. Values are expressed as the mean \pm standard deviation of at least three individual experiments. ns, not significant; ${ }^{*} \mathrm{P}<0.05$ vs. no starvation; ${ }^{*} \mathrm{P}<0.05,{ }^{* *} \mathrm{P}<0.01,{ }^{* * * *} \mathrm{P}<0.001$ vs. serum-starved group treated with neither TK nor $\mathrm{NH}_{4} \mathrm{Cl} ;{ }^{\star \star} \mathrm{P}<0.01,{ }^{\star \star \star} \mathrm{P}<0.001$ vs. serum-starved group treated with TK but not $\mathrm{NH}_{4} \mathrm{Cl}$. TK, tissue kallikrein; $\mathrm{LC}$, light chain.

A

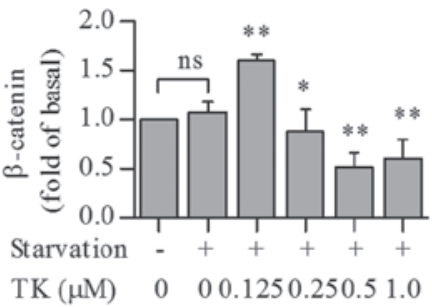

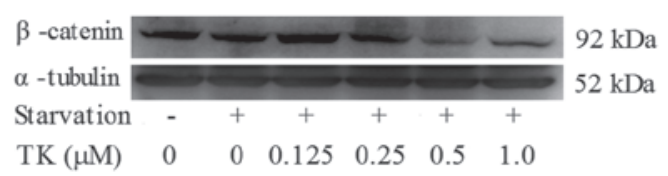

$\mathrm{TK}(\mu \mathrm{M}) \quad 0 \quad 00.1250 .250 .51 .0$
B

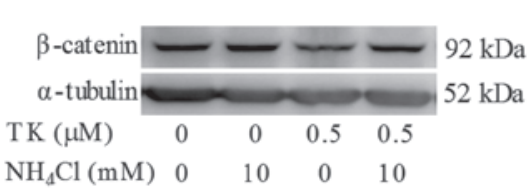

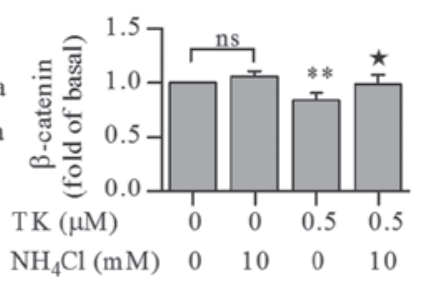

C

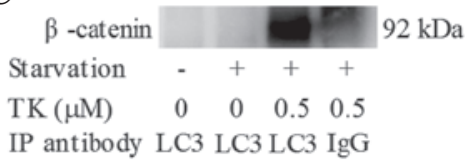

Figure 4. TK promotes autophagic degradation of $\beta$-catenin in serum-starved SH-SY5Y cells. (A) SH-SY5Y cells were pre-treated with 0.125-1.0 $\mu$ M TK and serum-starved for $12 \mathrm{~h}$. (B) SH-SY5Y cells were treated with $0.5 \mu \mathrm{M} \mathrm{TK}$ or in combination with $10 \mathrm{mM} \mathrm{NH}_{4} \mathrm{Cl}$ and serum-starved for $12 \mathrm{~h}$. $\beta$-catenin expression was detected by western-blot analysis with $\alpha$-tubulin used as the loading control. Values are expressed as the mean \pm standard deviation of at least three individual experiments. (C) SH-SY5Y cells were left untreated or treated with $0.5 \mu \mathrm{M} \mathrm{TK}$ and serum-starved for $8 \mathrm{~h}$. Co-immunoprecipitation was performed to detect the interaction between $\mathrm{LC} 3$ and $\beta$-catenin. ns, not significant; ${ }^{*} \mathrm{P}<0.05,{ }^{* * *} \mathrm{P}<0.01$ vs. serum-starved group treated with neither $\mathrm{TK}$ nor $\mathrm{NH}_{4} \mathrm{Cl}$. ${ }^{\star} \mathrm{P}<0.05$ vs. serum-starved group treated with TK but not $\mathrm{NH}_{4} \mathrm{Cl}$. IP, immunoprecipitation; TK, tissue kallikrein; LC light chain; IgG, immunoglobulin G.

The findings of the present study are consistent with a previous study, which reported that $\mathrm{Wnt} / \beta$-catenin represses autophagy and p62 expression, while $\beta$-catenin itself is targeted by autophagic clearance in autolysosomes upon induction of autophagy (11). Indeed, TK was shown to induce $\beta$-catenin downregulation, which was abrogated by $\mathrm{NH}_{4} \mathrm{Cl}$; furthermore, co-immunoprecipitation analysis revealed that TK stimulated the interaction between LC3 and $\beta$-catenin. These findings 
indicated that TK promoted the autophagic degradation of $\beta$-catenin to favor adaptation-promoting autophagy, thereby mitigating the deleterious consequences of continued proliferation and transcription during stress.

In the experimental model used in the present study, serum starvation as well as TK treatment increased the protein levels of p62, which however, is not necessarily indicative of blocked autophagic flux in this context $(8,11)$. Under starvation conditions, depletion of p62 has been reported to inhibit the recruitment of LC 3 to autophagosomes and the background level of LC3-II was shown to be elevated in cells overexpressing p62, indicating that autophagic activity is increased with increasing levels of p62 (21). In the present study, RT-qPCR analysis further suggested that the observed elevation of $\mathrm{p} 62$ protein was, at least partially, attributed to increased transcription. Furthermore, pre-treatment with the lysosome inhibitor $\mathrm{NH}_{4} \mathrm{Cl}$ in the presence as well as absence of TK led to a recovery of the levels of p62 and LC3-II, indicating normal autophagic flux.

In conclusion, the present study provided direct evidence that autophagy contributed to the pro-survival effects of TK in SH-SY5Y cells under serum starvation. TK initiated autophagy while maintaining normal autophagic flux. The present study enhanced the present understanding of key cellular processes affected by TK and revealed the potential use of TK for alleviating nutrient deprivation-induced stress conditions such as ischemic stroke. The underlying molecular mechanisms of the modulation of autophagy by TK require to be elucidated in future studies.

\section{Acknowledgements}

This work was supported by a grant from the National Natural Science Foundation of China (no. 81271295).

\section{References}

1. Tanida I: Autophagosome formation and molecular mechanism of autophagy. Antioxid Redox Signal 14: 2201-2214, 2011.

2. Bhoola KD, Figueroa CD and Worthy K: Bioregulation of kinins: Kallikreins, kininogens and kininases. Pharmacol Rev 44: 1-80, 1992.

3. Su J, Tang Y, Zhou H, Liu L and Dong Q: Tissue kallikrein protects neurons from hypoxia/reoxygenation-induced cell injury through Homerlb/c. Cell Signal 24: 2205-2215, 2012.

4. Wang Z, Han X, Cui M, Fang K, Lu Z and Dong Q: Tissue kallikrein protects rat hippocampal CA1 neurons against cerebral ischemia/reperfusion-induced injury through the B2R-Raf-MEK1/2-ERK1/2 pathway. J Neurosci Res 92: 651-657, 2014.

5. Tang Y, Shao Y, Su J, Zhou H, Liu L, Ren H and Dong Q: The protein therapy of kallikrein in cerebral ischemic reperfusion injury. Curr Med Chem 16: 4502-4510, 2009.
6. Papadakis M, Hadley G, Xilouri M, Hoyte LC, Nagel S McMenamin MM, Tsaknakis G, Watt SM, Drakesmith CW, Chen R, et al: Tsc1 (hamartin) confers neuroprotection against ischemia by inducing autophagy. Nat Med 19: 351-357, 2013.

7. Yuan G, Deng J, Wang T, Zhao C, Xu X, Wang P, Voltz JW, Edin ML, Xiao X, Chao L, et al: Tissue kallikrein reverses insulin resistance and attenuates nephropathy in diabetic rats by activation of phosphatidylinositol 3-kinase/protein kinaseB and adenosine 5'-monophosphate-activated protein kinase signaling pathways. Endocrinology 148: 2016-2026, 2007.

8. Kim JH, Hong SK, Wu PK, Richards AL, Jackson WT and Park JI: Raf/MEK/ERK can regulate cellular levels of LC3B and SQSTM1/p62 at expression levels. Exp Cell Res 327: 340-352, 2014.

9. Wang J, Whiteman MW, Lian H, Wang G, Singh A, Huang D and Denmark T: A non-canonical MEK/ERK signaling pathway regulates autophagy via regulating Beclin 1. J Biol Chem 284: 21412-21424, 2009.

10. Moon RT: Wnt/beta-catenin pathway. Sci STKE 2005: 2005.

11. Petherick KJ, Williams AC, Lane JD, Ordóñez-Morán P, Huelsken J, Collard TJ, Smartt HJ, Batson J, Malik K, Paraskeva C and Greenhough A: Autolysosomal $\beta$-catenin degradation regulates Wnt-autophagy-p62 crosstalk. EMBO J 32: 1903-1916, 2013.

12. Klionsky DJ, Abdalla FC, Abeliovich H, Abraham RT, Acevedo-Arozena A, Adeli K, Agholme L, Agnello M, Agostinis P, Aguirre-Ghiso JA, et al: Guidelines for the use and interpretation of assays for monitoring autophagy. Autophagy 8: 445-544, 2012.

13. Waeckel L, Potier L, Richer C, Roussel R, Bouby N and Alhenc-Gelas F: Pathophysiology of genetic deficiency in tissue kallikrein activity in mouse and man. Thromb Haemost 110: 476-483, 2013.

14. Chao J, Shen B, Gao L, Xia CF, Bledsoe G and Chao L: Tissue kallikrein in cardiovascular, cerebrovascular and renal diseases and skin wound healing. Biol Chem 391: 345-355, 2010.

15. Yao YY, Yin H, Shen B, Smith RS Jr, Liu Y, Gao L, Chao L and Chao J: Tissue kallikrein promotes neovascularization and improves cardiac function by the Akt-glycogen synthase kinase-3beta pathway. Cardiovasc Res 80: 354-364, 2008.

16. Lu Z, Yang Q, Cui M, Liu Y, Wang T, Zhao H and Dong Q: Tissue kallikrein induces SH-SY5Y cell proliferation via epidermal growth factor receptor and extracellular signal-regulated kinase1/2 pathway. Biochem Biophys Res Commun 446: 25-29, 2014.

17. Chong ZZ and Maiese K: Targeting WNT, protein kinase B and mitochondrial membrane integrity to foster cellular survival in the nervous system. Histol Histopathol 19: 495-504, 2004.

18. Heras-Sandoval D, Pérez-Rojas JM, Hernández-Damián J and Pedraza-Chaverri J: The role of PI3K/AKT/mTOR pathway in the modulation of autophagy and the clearance of protein aggregates in neurodegeneration. Cell Signal 26: 2694-2701, 2014.

19. Georgopoulos NT, Kirkwood LA and Southgate J: A novel bidirectional positive-feedback loop between Wnt- $\beta$-catenin and EGFR-ERK plays a role in context-specific modulation of epithelial tissue regeneration. J Cell Sci 127: 2967-2982, 2014.

20. Wei Y,Zou Z, Becker N, Anderson M, Sumpter R, Xiao G, Kinch L, Koduru P, Christudass CS, Veltri RW, et al: EGFR-mediated Beclin 1 phosphorylation in autophagy suppression, tumor progression, and tumor chemoresistance. Cell 154: 1269-1284, 2013.

21. Bjørkøy G, Lamark T, Brech A, Outzen H, Perander M, Overvatn A, Stenmark H and Johansen T: P62/SQSTM1 forms protein aggregates degraded by autophagy and has a protective effect on huntingtin-induced cell death. J Cell Biol 171: 603-614, 2005. 